\title{
Dispersion in simulation models of population growth and salt movement in the soil
}

\author{
J. Goudriaan \\ Department of Theoretical Production Ecology, Agricultural University, Wageningen, \\ the Netherlands
}

Accepted: 3 July 1973

\section{Summary}

The basic equations for mass transport of salt in the soil are analogous to those for development in a population. Both types of processes are modelled by compartmentalization. In such models with layers or classes an artificial dispersion is introduced with a coefficient $\mathrm{vB} / 2$, where $\mathrm{v}$ is the rate of transport or development and $\mathrm{B}$ the size of the compartment. Rectangular integration causes an additional dispersion with a coefficient $-v^{2} \Delta t / 2$, where $\triangle t$ is the time interval of integration. The artificial dispersion can be used to mimick the natural dispersion. Methods to control or prevent artificial dispersion are discussed in relation to the used integration method.

\section{List of symbols}

B thickness of layer, size of development class

C concentration of salt in a layer, number of individuals in a class

D diffusivity

F fraction of class to be shifted

$\mathrm{N}$ number of classes, or number of class

$\sigma \quad$ standard deviation in space or development with B as unit

$\sigma_{\mathrm{s}} \quad$ standard deviation in space

$\sigma_{\mathrm{d}} \quad$ standard deviation in development

$\sigma_{t} \quad$ standard deviation in time

$\mathrm{S}$ displacement or shift in space or development

$\mathrm{t}$ time

$t_{\mathrm{av}} \quad$ average time of shift or development up to a certain point

$v$ rate of water flow in the pores, rate of development

$v^{\prime} \quad$ rate of mass flow of water

$\mathrm{x} \quad$ space or development dimension

\section{Introduction}

If salt water infiltrates a column of soil at one end, a gradual increase in salt concentration can be noticed at the other end. The curve of the change in time of the salt concentration in the outflowing water is called the breakthrough curve. The shape of this 
curve can be explained by diffusion of salt in water, but for most salts or ions molecular diffusion is by no means sufficient to cause the observed degree of levelling. Some reasons of the increase of the apparent diffusivity are interactions between the soil and the salts, such as adsorption and chemical exchange. Perhaps the velocity of the liquid in different pores is different, and so some of the salt will be transported more rapidly than other portions. These and other effects that cause levelling of the front are summarized in the term natural dispersion.

A similar phenomenon can be noticed in the development of living organisms. If a number of seeds are sown simultaneously, that have an average germination time of 20 days, the germination times of the individual seeds will be scattered around the value of 20 days. The shape of the cumulative curve of germinated seeds against time will be similar to that of the breakthrough curve. The scatter in germination time can be reduced to a scatter in environmental conditions and to a scatter in individual properties of the seeds.

Any simulation model aiming at an explanation of the dispersion, either of development or of salt transport, should contain the reasons for it, of which some have been mentioned. It is also possible to give only a description of the dispersion in a simulation model. In all cases, there is still the pitfall of introducing an artificial dispersion just by the structure of the model itself. This applies to both soil and population models. The theory and the prevention or control of the artificial dispersion is the subject of this paper.

\section{Analogies of soil and population models}

It is usual in models of soil transport processes to divide the soil into a number of layers (de Wit \& van Keulen, 1972). The rate of water flow from one layer to another and the salt concentrations determine the quantity of salt transported. The natural dispersion can be allowed for by an apparent diffusion coefficient.

Most models describing development of plant organs or of animal populations are divided into development stages. Development is described as a transition from one development class to the next. The rate of this transition is proportional to the number of individuals in the class and to the rate of development.

There are the following analogies:

$\begin{array}{lll}\text { Symbol } & \text { Population model } & \text { Soil model } \\ \text { B } & \text { Size of development class } & \text { Thickness of layer } \\ \text { C } & \text { Number of individuals in } & \text { Concentration of salt } \\ & \text { a development class } & \text { in a layer } \\ \text { v } & \text { Rate of development } & \text { Rate of water flow } \\ \text { D } & \text { Diffusivity (not used) } & \text { Diffusivity }\end{array}$

\section{Artificial dispersion in the soil mode!}

The soil is assumed to be saturated with water and to have a porosity $\varepsilon$. There is a mass flow of water with velocity $v^{\prime}$ and the water in the pores flows with a velocity $v=$ $v^{\prime} / \varepsilon$. The salt concentration in the water is $C(x, t)$. The apparent diffusivity is $D$. In this case the differential equation for the rate of change of $\mathrm{C}$ under influence of water flow and diffusion is 
POPULATION GROWTH AND SALT MOVEMENT IN SOIL

$$
\frac{\delta \mathrm{C}}{\delta \mathrm{t}}=-\mathrm{v} \frac{\delta \mathrm{C}}{\delta \mathrm{x}}+\mathrm{D} \frac{\delta^{2} \mathrm{C}}{\delta \mathrm{x}^{2}}
$$

The term $-\mathrm{v} \frac{\delta \mathrm{C}}{\delta \mathrm{x}}$ causes the whole profile to shift unchanged with velocity $\mathrm{v}$, and the term $\mathrm{D} \frac{\delta^{2} \mathrm{C}}{\delta \mathrm{x}^{2}}$ levels the peaks and dips. The simplest way to study the effect of simulation on the degree of levelling is to put apparent diffusivity $D$ at zero. Then the profile should move unchanged. For the purpose of simulation the soil is divided into layers with thickness B. Within each layer the salt concentration is supposed to be uniform and equal to $C(I)$, when $I$ is the number of the layer. Eq. 1 is transformed into a differential equation for the salt concentration in layer $\mathrm{I}$ :

$$
\frac{\mathrm{dC}(\mathrm{I})}{\mathrm{dt}}=\mathrm{v} \underset{\mathrm{B}}{\mathrm{C}(\mathrm{I}-1)-\mathrm{C}(\mathrm{I})}
$$

The flow into a layer is the product of velocity and salt concentration in a preceding layer, the outflow equals the product of velocity and concentration in the same layer. The net flow is distributed over a thickness B and must therefore be divided by $\mathrm{B}$.

The inflow into a layer is not dependent on its own content, but on the content of the previous layer. The outflow, however, is proportional to its own content. If water with concentration zero flows into layer zero, this layer will loose salt proportional to the concentration in it. The differential equation for this situation is:

$$
\frac{\mathrm{dC}(0)}{\mathrm{dt}}=-\mathrm{vC}(0) / \mathrm{B}
$$

The solution is

$$
\mathrm{C}(0)=\mathrm{C}_{\mathrm{i}}(0) \mathrm{e}^{-\mathrm{vt} / \mathrm{B}}
$$

when $C_{i}(0)$ is the initial concentration in layer zero.

The inflow into the next layer is $\mathrm{vC}(0)$ and so the differential equation for layer number 1 is:

$$
\frac{\mathrm{dC}(1)}{\mathrm{dt}}=-\mathrm{vC}(1) / \mathrm{B}+\mathrm{v}_{\mathrm{i}}(0) \mathrm{e}^{-\mathrm{vt} / \mathrm{B} / \mathrm{B}}
$$

Taking the initial concentration zero for this layer, the solution is

$$
C(1)=\frac{v t}{B} C_{i}(0) e^{-v t / B}
$$

In the same way it follows that

$$
C(2)=1 / 2\left(\frac{v t}{B}\right)^{2} C_{i}(0) e^{-v t / B}
$$

Induction shows that the general solution is

$$
\mathrm{C}(\mathrm{N})=\sum_{\mathrm{I}=-\infty}^{\mathrm{N}} \mathrm{C}_{\mathrm{i}}(\mathrm{I})\left(\frac{\mathrm{vt}}{\mathrm{B}}\right)^{\mathrm{N}-\mathrm{I}}-\frac{1}{(\mathrm{~N}-\mathrm{I}) !} \mathrm{e}^{-\mathrm{v} t / \mathrm{B}}
$$

which can be checked by substitution in Eq. 2 . 
In the present case $C_{i}(I)$ is zero for $I \neq 0$. The expression for $C(N)$ then is

$$
C(N)=C_{i}(0)\left(\frac{v t}{B}\right)^{N} \frac{1}{\bar{N} !} e^{-v t / B}
$$

The same formula applies to the Poisson probability distribution with an expectation value vt/B (e.g. Moroney, 1951), if $C_{i}(0)$ is unity. The concentration in layer $N$ is equal to the probability of taking number $\mathrm{N}$ from a Poisson probability distribution, when the expectation value is vt/B. It can be shown that the average displacement of the salt given by

$$
\mathrm{S}_{\mathrm{av}}=\sum_{\mathrm{N}=0}^{\infty} \frac{\mathrm{C}(\mathrm{N})}{\mathrm{C}_{\mathrm{l}}(0)} \mathrm{N} \mathrm{B}
$$

equals

$$
\mathrm{S}_{\mathrm{av}}=\left(\frac{\mathrm{vt}}{\mathrm{B}}\right) \mathrm{B}
$$

similar as in a pipeline flow.

The degree of levelling of the peak can be described by the standard deviation $\sigma$. In a Poisson distribution it equals the square root of the expectation value.

Since the expectation value is $\left(\frac{\mathrm{vt}}{\mathrm{B}}\right)$ with $\mathrm{B}$ as unit, the standard deviation is

$$
\sigma=\left(\frac{\mathrm{vt}}{\mathrm{B}}\right)^{1 / 2}
$$

The levelling of the peak here is due to dividing into layers in the simulation model and not to an erroneous choice of the integration time interval, as integration is correct.

When the expectation value is large enough, the Poisson distribution may be substituted by a normal or Gauss distribution with the same standard deviation. The concentration in layer $\mathrm{N}$ being represented by

$$
\mathrm{C}(\mathrm{N})=\left.\mathrm{C}_{\mathrm{i}}(0) \frac{1}{2 \sigma^{2} \pi}\right|^{1 / 2} \mathrm{e}^{-(\mathrm{N}-\mathrm{vt} / \mathrm{B})^{2} /\left(2 \sigma^{2}\right)}
$$

Substitution of 12 gives

$$
C(N)=C_{i}(0)\left[\frac{B}{2 \pi v t}\right]^{-1 / 2} e^{\left((N-v t / B)^{2} B\right) /(2 v t)}
$$

This result due only to dividing in layers, is very similar to flattening under influence of diffusion. An amount of salt $\mathrm{BC}_{\mathrm{i}}$, the same as here, release at time zero in the plane with $\mathrm{x}$ is zero, displaced by water with velocity $\mathrm{v}$, and flowing out by diffusion $\mathrm{D}$, will be distributed at time $t$, when reflection can be neglected, as (Crank, 1957):

$$
\mathrm{C}=\mathrm{C}_{\mathrm{i}} \mathrm{B}\left|\frac{1}{4 \pi \mathrm{Dt}}\right|^{-1 / 2} \mathrm{e}^{(\lambda-\mathrm{v} t)^{2} /(4 \mathrm{D} t)}
$$

This equation is identical to Eq. 14 if

$$
\mathrm{D}=\mathrm{vB} / 2
$$


This means that this effect of a simulation model, called artificial or mathematical dispersion, can be represented as a diffusion. The apparent diffusion coefficient is proportional to the chosen size of the layers. Its effect is additive to the diffusion coeffecient already entered in a model.

An exception must be made for the time that mass flow has not moved further than a few layers. Transition from a Poisson distribution to a normal distribution is not allowed, then. The exact effect of the model is given in Eq. 8.

\section{The method of the Taylor expansion}

In 1955 Glueckauf also arrived at Eq. 16 by a simpler analysis. He approximated the concentration profile at a certain moment by a Taylor series expansion to $\mathrm{x}$ :

$$
C(I-1)=C(I)-B \frac{d C}{d x}+\frac{B^{2}}{2 !} \frac{d^{2} C}{d^{2}}-\frac{B^{3}}{3 !} \frac{d^{3} C}{d^{3}} \ldots
$$

By substitution into Eq. 2 he obtained:

$$
\frac{\delta \mathrm{C}(\mathrm{I})}{\delta \mathrm{t}}=-\mathrm{v} \frac{\delta \mathrm{C}}{-\mathrm{x}}+\frac{\mathrm{vB}}{2 !} \frac{\delta^{2} \mathrm{C}}{\delta \mathrm{x}^{2}}+\frac{\mathrm{vB}^{2}}{3 !} \frac{\delta^{3} \mathrm{C}}{\delta \mathrm{x}^{3}} \cdots
$$

The terms of the third and higher order are neglected. Provided $D$ equals vB/2, Eq. 18 is similar to Eq. 1. This is a result similar to that in the previous paragraph. That method, however, is more general and can also describe the process at the beginning, wherein an infinite Taylor series is required. The derivation of Eq. 18 was given more detailed by Glueckauf (1955).

Up to this point continuity was assumed with respect to time, a condition surely met if a simulation language is used with a Runge Kutta method with self-adapting time interval, and also in analog models.

If, however, the rectangular integration method is used, slicing in time has an influence similar to slicing in space. The value of $\mathrm{C}(\mathrm{I})_{t+\Delta t}$ can be written as a Taylor series with $\triangle \mathrm{t}$ as interval.

$$
\mathrm{C}_{\Delta \mathrm{t}}=\mathrm{C}+\frac{\mathrm{dC}}{\mathrm{dt}} \Delta \mathrm{t}+\frac{\mathrm{d}^{2} \mathrm{C}}{\mathrm{dt}^{2}} \frac{(\triangle \mathrm{t})^{2}}{2 !}
$$

or

$$
\frac{C_{\Delta t}-C}{\Delta t}=\frac{d C}{d t}+\frac{d=C}{d^{2}} \frac{\Delta t}{2 !}
$$

This expression indicates the value of the first derivative as it should be used in a rectangular method to arrive at the right value. The term $\Delta t \frac{\mathrm{dt}^{2}}{\mathrm{~d}^{2}} \mathrm{C}$, however, is usually omitted. As $\mathrm{dx}=\mathrm{v} \mathrm{dt}$ this term equals $\mathrm{v}^{2} \frac{\Delta \mathrm{t}}{2 !} \frac{\mathrm{dx}^{2}}{\mathrm{~d}^{2} \mathrm{C}}$. Therefore, an additional diffusion is introduced with a diffusion coefficient $-v^{2} \triangle t / 2$. In this case the sign is negative!

Likewise, it can be shown that no additional diffusion due to the time interval is introduced by the use of the trapezium or higher order integration methods. 


\section{Dispersion in space, and dispersion in time}

There are two equivalent methods to describe the degree of dispersion. The common method in infiltration experiments is a representation by the breakthrough curve. This

\section{Breakthrough curve at distance 10}

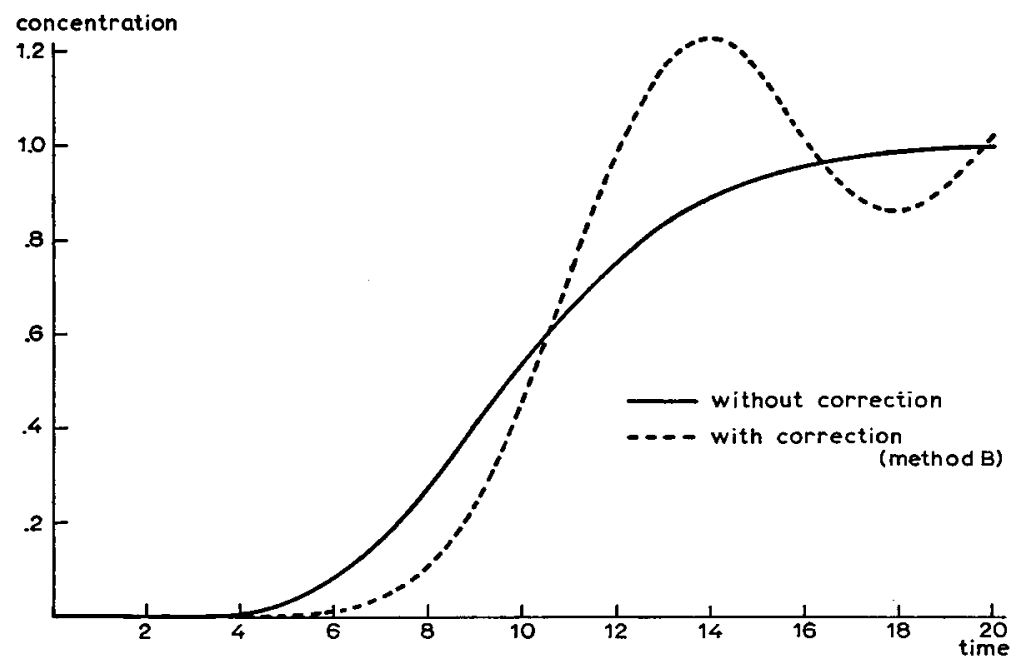

Fig. 1. The breakthrough curve at distance 10, showing the artificial dispersion, and the influence of the correction by method $\mathbf{B}$.

$$
\text { Distribution of concentration at time } 10
$$

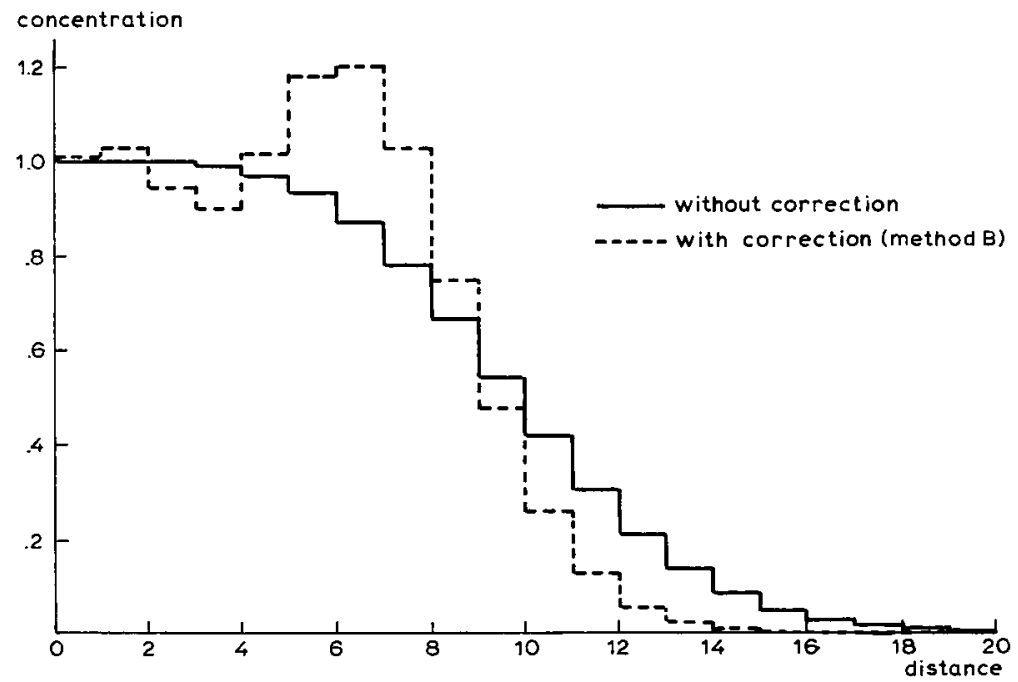

Fig. 2. Cross section through the profile at time 10 nuder the same circumstances. 
gives the course of the salt concentration in time at one fixed location (Fig. 1). In the alternative method a cross-section of the profile is made at one moment and the distribution of the concentration in space is taken as a measure for the dispersion (Fig. 2). From Eq. 9 the following relations can be derived. The average time of outflow from layer $\mathrm{N}$ is equal to:

$$
\mathrm{t}_{\mathrm{av}}(\mathrm{N})=\frac{\int_{0}^{\infty} \mathrm{t} \mathrm{C}(\mathrm{N}) \mathrm{dt}}{\int_{0}^{\infty} \mathrm{C}(\mathrm{N}) \mathrm{dt}}
$$

Integration shows that $\mathrm{t}_{\mathrm{av}}(\mathrm{N})$ equals

$$
\mathrm{t}_{\mathrm{av}}(\mathrm{N})=(\mathrm{N}+1) \mathrm{B} / \mathrm{v}
$$

where $\mathrm{N}+1$ is the number of layers. This is the same value as in case of a pipe line flow, and so the average time of outflow is unaffected by the artificial dispersion. The standard deviation of the time of outflow from layer $\mathrm{N}$ is:

$$
\sigma_{\mathrm{t}}{ }^{2}=\frac{\int_{0}^{\infty}\left(\mathrm{t}_{\mathrm{av}}-\mathrm{t}\right)^{2} \mathrm{C}(\mathrm{N}) \mathrm{dt}}{\int_{0}^{\infty} \mathrm{C}(\mathrm{N}) \mathrm{dt}}
$$

Again integration shows that

$$
\sigma_{\mathrm{t}}^{2}(\mathrm{~N})=\mathrm{t}_{\mathrm{av}} \mathrm{B} / \mathrm{v}
$$

Eq. 24 can also be written as:

$$
\sigma_{\mathrm{t}}^{2}(\mathrm{~N})=\mathrm{t}_{\mathrm{av}}{ }^{2} / \mathrm{N}
$$

if counting of $\mathrm{N}$ starts with 1 rather than zero.

Eq. 12 gives $\sigma$ (without subscript) in units of the size of the layer, B. Expressing it in the proper unit for space, it must be multiplied by $B$. If then time $t$ is replaced by the average time of outflow $t_{a v}$, the dispersion in space $\sigma_{s}$ appears to be equal to the product of $\mathrm{v}$ and the dispersion in time $\sigma_{\mathrm{t}}$, given by Eq. 24 , so that:

$$
\sigma_{\mathrm{s}}=\mathrm{v} \sigma_{\mathrm{t}}
$$

This conclusion can be extended to the normal distribution as given by Eq. 13, 14 and 15 under condition of Eq. 16.

\section{Artificial dispersion in a population model}

Largely the same equations can be employed as in the soil model. The population is divided in $\mathrm{N}$ development classes, each covering a range of development. Development here replaces space in the soil model. The flow rate from one class to the next equals the amount present in the first class, multiplied by the rate of development (v) divided by the size of the class expressed in development units (B). The definition of the development unit raises a problem which will be discussed later on. Without deaths, 
emigrations or immigrations occurring the differential equation for the amount in class $\mathrm{N}$ is:

$$
\frac{\mathrm{dH}(\mathrm{N})}{\mathrm{dt}}=\mathrm{v} \frac{\mathrm{H}(\mathrm{N}-1)-\mathrm{H}(\mathrm{N})}{\mathrm{B}}
$$

This equation conforms to Eq. 2 for the concentration in a soil layer. Consequently, the same conclusions are valid for a population model of this kind. An interesting result is the value of the standard deviation $\sigma$ in this case. According to Eq. 12:

$$
\sigma=\left(\frac{\mathrm{vt}}{\mathrm{B}}\right)^{1 / 2}
$$

in which $t$ is the age at which the dispersion is considered.

The unit of $\sigma$ is the size of a class, so that $\sigma$ in development units is

$$
\sigma_{\mathrm{d}}=\mathrm{B}\left(\begin{array}{l}
\mathrm{vt} \\
\mathrm{B}
\end{array}\right)^{1 / 2}
$$

or

$$
\sigma_{\mathrm{d}}=\mathrm{vt}\left(\frac{\mathrm{B}}{\mathrm{vt}}\right)^{1 / 2}
$$

or

$$
\sigma_{\mathrm{d}}=\mathrm{vt} / \mathrm{N}^{1 / 2}
$$

According to Eq. 26, a dispersion in time of the moments of outflow equals then:

$$
\sigma=\mathfrak{t}_{\mathrm{av}} / \mathrm{N}^{1 / 2}
$$

where $t_{a v}$ is the average time of outflow. In other words, the time $t$ in Eq. 31, the moment of taking the cross-section, is replaced by the average time of outflow. In case of a pipe line flow

$$
\mathbf{t}_{\mathrm{av}}=\mathrm{NB} / \mathrm{v}
$$

when $\mathbf{N}$ is the number of classes. This value may also be used in Eq. 31.

When there is a measured value of the standard deviation in, e.g. the moments of germination of seeds, the number of development classes can be adapted, so that the measured disperson is matched by the artificial dispersion. This number is given by Eq. 33:

$$
\mathrm{N}=\left(\mathrm{t}_{\mathrm{av}} / \sigma_{\mathrm{t}}\right)^{2}
$$

Increasing the number of stages decreases the standard deviation. Sometimes the standard deviation is so small that the number of classes to be used is impracticably large. Here, another method should be used, which will be discussed further in this paper.

This example shows that artificial dispersion can sometimes be efficiently used to imitate natural dispersion. At other times, however, it is preferable to suppress this phenomenon.

The size of a class should be expressed in units of development, and the rate of 
development in development units per time. A clearly defined unit of development can be found in, for example, the development of many insect species. There are four development stages: egg, larve, pupa and adult. Each of these stages can be given one unit of development, thus the entire range consists of four units of development. The problem is, that the average residence periods in the different stages vary. This means that the rate of development, defined as the size of a development class (here unity) over average residence time varies during development, dependent on the stage of development. The above derived formulas for $\sigma$ do not apply to this situation. Therefore another definition of development class may be useful, especially when the formula is to be used for $\sigma$.

At first, some standard conditions will be defined, under which development is closely followed. Then, the entire range of development is divided into basic classes equal in age, each covering a week, for instance. The rate of development is then equal to unity under the standard conditions. At a lower temperature it will generally take longer than one week to proceed to the next characteristic development criterion than under the standard conditions, and so the rate of development will be less than unity. The main condition for the applicability of this method is, however, that the influence of external conditions on the rate of development is the same for all development stages. The ratio between $\sigma_{\mathrm{t}}$ and the considered lifetime is constant then and independent on external conditions.

\section{Control of dispersion}

Five methods will be discussed to control artificial dispersion in dynamic simulation models. The first two methods that will be discussed can be used in analog simulation and in digital simulation when more sophisticated methods of integration are used. These methods suppress artificial dispersion but do not eliminate it. The other three methods can be used in digital simulation when the simple rectèlinear method of integration is used. The first of these has the disadvantage that the finite time interval of integration is misused for controlling dispersion by adapting its size. The second one eliminates dispersion completely and simulates pipe line flow. The third one, however, puts artificial dispersion to good use and allows to simulate dispersion phenomena independent of the number of classes or layers that are used and the rate of development or transport. This method is strongly recommended for use in digital and hybrid simulation.

\section{Method A: Increasing the number of layers}

As all phenomena of this sort are proportional to the size of the layer or development class a good deal of suppression can be obtained by reducing their size, thereby increasing their number. As it can be shown that the computation time is roughly proportional to the third power of this number, obviously practical limits in this direction are very soon reached.

\section{Method B: Averaging the contents of adjacent layers}

Large suppression can be easily obtained by a different formulation of the equations for the flow from one layer of group to the next one. As expressed by Eq. 2 this flow is proportional to the content of the preceding layer. In the alternative formulation this flow is proportional to the average between the contents of the adjacent layers. In the 
formula for the net flow the content of the layer itself is not taken into account, and the first derivative of $\mathrm{C}(\mathrm{I})$ is:

$$
\frac{\mathrm{dC}(\mathrm{I})}{\mathrm{dt}}=\mathrm{v} \frac{\mathrm{C}(\mathrm{I}-1)-\mathrm{C}(\mathrm{I}+1)}{2 \mathrm{~B}}
$$

According to the Taylor expansion:

$$
C(I+1)=C(I)+B \frac{d C}{d x}+\frac{B^{2} d^{2} C}{2 ! d x^{2}}+\frac{B^{3} d^{3} C}{3 ! d x^{3}} \cdots
$$

The Taylor expansion for $\mathrm{C}(\mathrm{I}-1)$ is given in Eq. 17, and substitution of Eq. 17 and 35 into Eq. 34 gives:

$$
\frac{\delta \mathrm{C}(\mathrm{I})}{\delta \mathrm{t}}=-\mathrm{v} \frac{\delta \mathrm{C}}{\delta \mathrm{x}}-\mathrm{v} \frac{\mathrm{B}^{3} \delta^{3} \mathrm{C}}{3 ! \delta \mathrm{x}^{3}} \ldots
$$

All even terms cancel, so that also the second-order term has gone. Therefore the artificial dispersion introduced by simulation is removed. In Fig. 1 a comparison is given between simulation of a breakthrough curve employing Eq. 2 and Eq. 34. The flattening is reduced for a good deal. The remaining distortion is due to the third, fifth and higher order terms that still remain. It is stressed here that this method is exactly the same as adding to the system a diffusion with a coefficient $-\mathrm{vB} / 2$, when Eq. 2 is used. Eq. 2 is transformed by this manipulation into Eq. 34 .

It is possible to eliminate higher-order terms in Eq. 36 by using more complicated formulas than Eq. 34, but this proved not to be worthwhile. A consequence of the higher-order terms is that in extreme situations, like a stepwise change to zero, negative values may be canculated. That this does not occur when Eq. 2 is used is only because this effect is overruled by the artificial dispersion. Similarly, when the physical diffusion is larger than $\mathrm{vB} / 2$, it will overrule the distortion. In view of this $B$ should not be chosen larger than $2 \mathrm{D} / \mathrm{v}$, apart from other possible restrictions for the size of $\mathrm{B}$.

Method C: Adaptation of the time interval of integration

If the rectangular method is employed in numerical integration it must be taken into account that an additional diffusivity of $-v^{2} \triangle t / 2$ is introduced (section 'The method of the Taylor expansion'), irrespective of using Eq. 34 or 2 for the first derivative. This leads to the conclusion that the rectangular method must be avoided, if method $\mathrm{B}$ is used.

If Eq. 2 is employed for the first derivative, the combined effect of layer size and time interval is an artificial dispersion with the coefficient $\frac{v}{2}(B-v \triangle t)$. This may have implications if the artificial dispersion is deliberately used to account for some sort of natural dispersion. The simplest way is to avoid the rectangular method, the more complicated way is to consider both effects and to choose the right values of $B$ and $\Delta t$ in combinaton.

From the equality of Eq. 13 and 15 can be derived that the standard deviation equals

$$
\sigma^{2}=2 \mathrm{Dt} / \mathrm{B}^{2}
$$

or

$$
\sigma^{2}=v(B-v \Delta t) t / B^{2}
$$


When $\sigma$ is expressed in the unit of time $\sigma_{\mathrm{t}}$ is:

$$
\sigma_{\mathrm{t}}^{2}=(B-v \Delta t) t / v
$$

In the population model $\mathrm{v}$ is equal to unity, and $\mathrm{t}$ is denoted by $\mathrm{t}_{\mathrm{av}}$, the age at the considered point. Then is $\sigma_{\mathrm{t}}$

$$
\sigma_{\mathrm{t}}^{2}=\left(\mathrm{t}_{\mathrm{av}} / \mathrm{N}-\triangle \mathrm{t}\right) \mathrm{t}_{\mathrm{av}}
$$

When this is written as an expression for $\mathrm{N}$ it reads

$$
\mathrm{N}=\mathrm{t}_{\mathrm{av}}{ }^{2} /\left(\sigma_{\mathrm{t}}^{2}+\Delta \mathrm{t}_{\mathrm{av}}\right)
$$

When $\sigma_{\mathrm{t}}$ is known, a reasonable value of $\mathrm{N}$ and $\Delta \mathrm{t}$ can be chosen. This is especially important, if there is a risk of $\mathrm{N}$ being too large. A principle and large objection against this method is that the time interval for integration is part of the structure of he system. Method E overcomes this objection.

In the matrix method for population models as designed by Leslie in $1945 \triangle t$ is made equal to $t_{a v} / N$, or $B / v$. The integration taking place in every time interval is here replaced by multiplication of a vector, composed of the contents of the different age classes, by a matrix in which the death rate and the birth rate are accounted for. The special features and merits of this method are describer by Leslie (1945) and Usher \& Williamson (1970). This method is, however, much less flexible than a regular simulation method.

\section{Method D: Boxcar train with complete repeated shift}

In numerical integration, the combined effect of layer size and time interval in dispersion cancel against each other if the rectilinear method of Euler is used and the time interval of integration is set equal to $\mathrm{B} / \mathrm{v}$. In that case there is no artificial dispersion. A refined application of this principle is the complete repeated shift. In that case the time interval of integration may be chosen small enough to satisfy the criteria for integration of any continuous processes in other parts of a model. However, once every average residence time $(\mathrm{RT}=\mathrm{B} / \mathrm{v})$ all the contents of the layers or development classes are shifted one place. It is impossible to present the method in differential equation notation. Instead it is presented in a Continuous System Simulation Language (CSSL) type notation as follows:

$$
\begin{aligned}
& \mathrm{H}_{1}=\mathrm{INTGRL}\left(\mathrm{HO}_{\mathrm{I}}, \mathrm{RATE}+\mathrm{PUSH} *\left(\mathrm{H}_{\mathrm{I}-1}-\mathrm{H}_{\mathrm{I}}\right) / \mathrm{DELT}\right) \\
& \mathrm{PUSH}=\mathrm{INSW}(\mathrm{A}-1,0,1) \\
& \mathrm{A}=\mathrm{INTGRL}(0 ., 1 / \mathrm{RT}-\mathrm{PUSH} / \mathrm{DELT})
\end{aligned}
$$

The first argument of the INTGRL 'function' is the initial amount or integration constant, and the second argument the rate of change of $H_{I}$. The INSW function keeps PUSH at zero, except at the moment when A-1 is larger than 0 , when PUSH is made equal to 1 . DELT stands for $\triangle \mathrm{t}$.

By using the rectilinear method of integration $\mathrm{H}_{\mathrm{I}, \mathrm{t}+\Delta \mathrm{t}}=\mathrm{H}_{\mathrm{I}, \mathrm{t}}+\mathrm{PUSH} *\left(\mathrm{H}_{\mathrm{I}-1, \mathrm{t}}-\right.$ $\mathrm{H}_{\mathrm{I}, \mathrm{t}} \mathrm{g} / \mathrm{DELT} * \triangle \mathrm{t}$ it is seen that the whole contents of the layer or development classes are shifted to the next when PUSH equals 1 . At the same time the accumulator $A$ is decreased by 1 , so that PUSH is zero again, until A exceeds one.

The residence time RT is of course a variable. In a population model RATE is the death, immigration and emigration rate and also the birth rate in the first development 
class. In the soil model RATE stands for adsorptive exchange and chemical reaction rates and also the rate of inflow in the first layer. By using this boxcar train, dispersion of the contents of one class or layer into the other is completely prevented and a pipe line flow is simulated. Since it allows also a free choice of the interval of integration and a variable residence time, this method is very much preferred above Method B or C.

Method E: Boxcar train with fractional repeated shift

Along a similar line a method may be developed which controls and puts artificial dispersion to good use and allows nevertheless a free choice of the integration interval. In this case not the whole content of a class or a layer is shifted every time when a full average residence time per class is passed, but only a fraction $F$ of the contents every time when a fraction $F$ of the residence time is passed. The flow through the compartments is handled in the same way as in the case of a rectilinear integration with time interval equal to $\mathrm{F}^{*} \mathrm{R}$ T. Therefore the formula which allows to express the fraction $\mathrm{F}$ in the number of classes $(\mathrm{N})$, the desired standard deviation $\left(\sigma_{t}\right)$ and the total residence time in the $N$ classes $\left(t_{a v}=N * R T\right)$ are given in Eq. 40 and 41 . Replacing $\triangle t$ by $F^{*} \mathrm{RT}$ and writing $\mathrm{F}$ explicite it is found that

$$
\mathrm{F}=1 \text {. }-\mathrm{N} \sigma_{\mathrm{t}}{ }^{2 / \mathrm{t}^{2}{ }_{\mathrm{av}}}
$$

Since the formula is applied in case of numerical, rectilinear integration the minimum value of $F$ equals DELT/RT. Whenever the calculated $F$ is smaller, this means that a too large number of classes was chosen. This fractional repeated shift is presented in a CSSL like notation as follows.

$$
\begin{aligned}
& \mathrm{H}_{\mathrm{I}}=\mathrm{INTGRL}\left(\mathrm{HO}_{\mathrm{I}}, \mathrm{RATE}+\mathrm{PUSH} * \mathrm{~F} *\left(\mathrm{H}_{\mathrm{I}-1}-\mathrm{H}_{\mathrm{I}}\right) / \mathrm{DELT}\right) \\
& \mathrm{PUSH}=\mathrm{INSW}(\mathrm{A}-1,0,1) \\
& \mathrm{A}=\mathrm{INTGRL}(0,1 /(\mathrm{F} * \mathrm{RT})-\mathrm{PUSH} / \mathrm{DELT}) \\
& \mathrm{F}=\text { AMAX1(DELT } / \mathrm{RT}, 1-\mathrm{N} *(\mathrm{SIGMA} / \mathrm{TAV}) * 2) \\
& \mathrm{RT}=\mathrm{TAV} / \mathrm{N}
\end{aligned}
$$

in which AMAX1 is a function that takes the maximum of the two arguments. This method can be applied in situations where the residence time and both the residence time and sigma are variables.

In the following scheme some of the methods are summarized.

Integration method

Purpose

Prevention of

dispersion

Control of

dispersion
Rectilinear

Complete shift

$\mathbf{F}=1$.

Method D

Fractional shift

$F=1 .-\mathrm{N}\left(\frac{\sigma_{\mathrm{t}}}{\mathrm{t}_{\mathrm{av}}}\right)^{2}$

Method E
Other

Corrected flow equation Method B

$N=\left(\stackrel{t_{a v}}{\sigma_{\mathrm{t}}}\right)^{2}$ 


\section{References}

Crank, J., 1956. Mathematics of diffusion. University Press, Oxford.

Ferrari, Th. S. \& J. L. Cuperus, 1973. Dynamic simulation of vertical non-adsorbed anion transport. Pl. Soil 38: 425-438.

Glueckauf, E., 1955. The theoretical plate concept in column separations, Trans. Faraday Soc. 51: 34. Leslie, P. H., 1945. On the use of matrices in certain population mathematics. Biometrika 33: 183-212. Moroney, M. J., 1951. Facts from figures. Penguin books, Harmondsworth.

Usher, M. B. \& M. H. Williamson, 1970. A deterministic matrix model for handling the birth, death and migration processes of spatially distributed populations. Biometrics 26 (1).

Wit, C. T. de \& H. van Keulen, 1972. Simulation of transpott processes in soils. Simulation Monographs No 1. Pudoc, Wageningen. 